\title{
Preventive Care in Chronic Liver Disease
}

\author{
Thomas R. Riley, MD, Jill P. Smith, MD
}

\begin{abstract}
OBJECTIVE: To identify preventive care measures that are appropriate for and specific to patients with chronic liver disease and to provide recommendations and information that can be shared with patients.
\end{abstract}

MEASUREMENTS: A review of the literature was undertaken using MEDLINE from 1970 to present. Priority was given to randomized controlled studies, but case reports, case-control studies, and reviews were included.

MAIN RESULTS: Evidence for the avoidance of alcohol and other toxic substances, immunizations, and dietary modifications for chronic liver disease is summarized. In addition, measures that are effective in the mitigation of the complications of cirrhosis are reviewed.

CONCLUSIONS: Preventive care can play an important role in patients with chronic liver diseases. Based on the existing data, the preventive strategies of alcohol avoidance, hepatitis vaccination, avoidance of NSAIDs nonsteroidal anti-inflammatory drugs, iron supplementation when appropriate, and a low-fat diet are prudent in patients with chronic liver disease. Once cirrhosis develops, screening for hepatocellular cancer with $\alpha$-fetoprotein testing and ultrasound, and screening for varices by endoscopy are justified.

KEY WORDS: liver diseases; prevention; hepatitis; cirrhosis; immunization; diet.

J GEN INTERN MED 1999;14:699-704.

C hronic liver disease is the tenth leading cause of death in the United States with more than 25,000 deaths annually according to federal statistics. There are an estimated 4 million known cases of hepatitis $\mathrm{C}$ in the United States; some of these patients present with cirrhosis or will eventually become cirrhotic. ${ }^{1}$ Our current ability to treat hepatitis $\mathrm{C}$ and eradicate the infection has been disappointing. ${ }^{2}$ Other chronic liver diseases that have no treatments include $\alpha_{1}$-antitrypsin deficiency, cryptogenic hepatitis, and primary sclerosing cholangitis.

Received from the Department of Medicine, Section of Gastroenterology and Hepatology, Penn State Geisinger Health System, Hershey, Pa (TRR, JPS).

Address correspondence and reprint requests to Dr. Riley: Milton S. Hershey Medical Center, M.C. H045, P.O. Box 850, Hershey, PA 17033-0850.
Some liver diseases, such as primary biliary cirrhosis, have treatments that delay progression but do not halt the liver injury. ${ }^{3}$ Many of these diseases progress slowly from hepatitis to cirrhosis, often over 20 to 30 years.

With an inability to cure the primary disease process, it becomes important to prevent further exacerbation and optimize the length of time between hepatitis and cirrhosis. Once cirrhosis occurs, nothing currently can be done to reverse the process. Individuals with early cirrhosis may live on average 10 to 15 years before liver transplantation or death. ${ }^{4}$ Preventive strategies can maximize this time by avoiding further damage and mitigating comorbidity. This review will discuss preventive care methods that have been shown to be effective or have a scientific rationale in the setting of chronic liver diseases, in both the hepatitis and cirrhotic stages.

\section{METHODS}

An outline of relevant topics was made. A systematic review of the literature was undertaken using electronic search techniques of MEDLINE from 1970 to the present. Priority was given to randomized controlled trials, but reviews and case-control studies were utilized as well.

\section{RESULTS}

\section{Alcohol Consumption}

Alcohol consumption of more than four drinks (48 g) daily is associated with an increased risk of cirrhosis, liver cancer, and premature death. ${ }^{5}$ The types of injury to the liver directly related to alcohol intake include acute alcoholic hepatitis, fatty infiltration, and cirrhosis. ${ }^{6}$ There is little information available to determine what level of alcohol consumption might be safe in those with a chronic liver condition. In a recent report, Corrao et al. examined the combined effect of hepatitis $\mathrm{C}$ and alcohol on the development of symptomatic cirrhosis. ${ }^{7}$ They found that alcohol had a synergistic, not merely additive, role in the development of cirrhosis in patients infected with hepatitis C. The group with a moderate level of alcohol consumption carried an increased risk of developing cirrhosis. ${ }^{7}$

Because the safe level of alcohol consumption in chronic liver disease is not known, it is prudent to advise abstinence. When moderate to heavy alcohol use is present, abstinence is likely to be the most important intervention 
for prolonging the course and delaying the progression of chronic liver diseases toward cirrhosis. Alcohol has a strong addictive potential, and abstinence can be difficult. Support should be provided in many of the cases where heavy use is present. Examples of effective support are Alcoholics Anonymous, inpatient and outpatient rehabilitation programs, and individual counseling. ${ }^{8}$

\section{Immunization}

Case reports have raised concerns about the risks of superinfection with the hepatitis viruses in those with chronic liver diseases. Both hepatitis A and B are reported to cause more virulent acute infections when a chronic liver condition exists. ${ }^{9-12}$ Vento et al. followed prospectively 163 patients with chronic hepatitis B and 432 patients with hepatitis C. Their study showed a substantial risk of fulminant hepatic failure and death with hepatitis A superinfection, in particular in those with chronic hepatitis C. Ordinarily, fewer than 1 in 1,000 cases of hepatitis A are fatal. In this series, when chronic hepatitis $\mathrm{C}$ was present, $35 \%$ of cases were fatal (a risk of death 350-fold above normal). ${ }^{13}$ In another large series of 115,551 cases of acute hepatitis A, there were 381 deaths. Of these deaths, 107 (28\%) were in patients with chronic liver disease. ${ }^{11}$ These data demonstrate the importance of testing first for exposure to the hepatitis viruses by measuring hepatitis A total antibody as well as hepatitis B core and surface antibodies in all those with chronic liver disease. If no immunity is found, then the hepatitis A vaccine (two-part series) and the hepatitis B vaccine (three-part series) should be administered. Both vaccines can be given safely in opposite deltoids at the same time; the hepatitis $B$ vaccine should be given 1 month later for the second in the series, and both vaccines should be administered again for completion 6 months later. ${ }^{14-16}$

A study by Keeffe et al. recently described the safety and efficacy of vaccination in patients with chronic liver disease. In all groups studied, including hepatitis B, hepatitis $\mathrm{C}$, and chronic liver disease patients, an overall $94 \%$ seroconversion rate after vaccination with inactivated hepatitis A vaccine was achieved. In the chronic hepatitis C patients in this study, hepatitis $B$ vaccination led to $100 \%$ seroconversion with hepatitis B vaccine series. ${ }^{17}$ These data are important because chronic illness and immunodeficiency have been shown in some instances to decrease vaccine seroconversion success rates. ${ }^{18,19}$ No vaccination currently is available for clinical use to protect against the acquisition of the hepatitis $\mathrm{C}$ virus. Pneumovax is also recommended as a one-time vaccination in all patients with cirrhosis. The rationale for this recommendation is to prevent episodes of pneumococcal spontaneous bacterial peritonitis (SBP) given that Streptococcus pneumonia is the third most common isolate, and to prevent pneumococcal pneumonia in a group that would tolerate infection poorly. ${ }^{20}$ All patients with cirrhosis should receive yearly influenza vaccine, given the higher risk of mortality should they contract influenza. ${ }^{21}$

\section{Potentially Liver-Toxic Medications, Vitamins, Herbs, and Minerals}

Most medications and ingested substances are altered chemically as they pass through the liver-a mechanism for clearance, detoxification, and excretion. Because of its central role in metabolic processing, the liver is vulnerable to toxicity from drugs and vitamins. ${ }^{22}$ In the setting of chronic liver diseases, liver function may variably be affected. Although most medications can be given safely to patients with chronic liver disease, altered liver functions may enhance or predispose patients to toxicity. In addition to a predisposition to medication toxicity, patients with chronic liver disease may be more affected by idiosyncratic reactions.

To avoid medication toxicity in patients with chronic liver disease, medications should be used judiciously. Each required medication should be evaluated for possible liver toxicity. If liver toxicity has been reported, an alternative with less or no adverse hepatic effects should be sought. If no options for safer alternatives exist, then the medication should be started under close observation. The patient with chronic liver disease should be made aware of hepatic-related symptoms, such as jaundice, pruritus, anorexia, fatigue, and right upper quadrant abdominal pain. Liver injury tests, including determination of transaminase, total bilirubin, and alkaline phosphatase levels, can be conducted at baseline, every 2 weeks for a month, monthly for 3 months, then every 3 months while the patient uses the potential hepatotoxin. If liver injury tests show levels increased more than two to three times the baseline, or if liver-related symptoms develop, the medication should be discontinued. ${ }^{23}$ When a question arises about the safety of a medication in an individual with chronic liver disease, a hepatologist's input may be helpful.

Pain medications are widely used, with both prescription and over-the-counter preparations available. Nonsteroidal anti-inflammatory drugs (NSAIDs), the largest class of medications used for pain and headache, can cause idiosyncratic liver toxicity. ${ }^{24} \mathrm{~A}$ recent report by Riley and Smith described three cases in which over-the-counter ibuprofen use in patients with hepatitis $\mathrm{C}$ resulted in a greater than 10-fold rise in the transaminase levels. ${ }^{25}$ In one of the cases, a rechallenge occurred leading to a second and equal rise in the transaminase levels. By the nature of these unpredictable (idiosyncratic) reactions, NSAIDs convey risk whenever they are used in the setting of chronic liver disease. In cirrhotic patients in whom there already is impaired coagulation, NSAIDs are known to increase the tendency for bleeding by inhibiting platelet function. They also decrease renal prostaglandins. This is important in cirrhotic patients, who are more dependent on prostaglandin-mediated blood flow. Because NSAIDs 
greatly decrease renal blood flow by afferent arteriolar vasoconstriction in cirrhotics, they decrease glomerulofiltration rates, which can lead to renal failure. ${ }^{26}$

For these reasons, NSAIDs should be avoided in patients with chronic liver diseases. When pain medications are required, preference should be given to acetaminophen at a dose of up to $2 \mathrm{~g}$ per day. Acetaminophen leads to an intrinsic, dose-dependent, and predictable toxicity to the liver. There are cases of toxicity reported even at acetaminophen doses below $4 \mathrm{~g}$ per day (therapeutic misadventure), usually associated with ethanol ingestion or starvation. $^{27}$

Vitamins and "alternative" natural remedies are commonly consumed in the United States with annual sales over $\$ 1.6$ billion. In informal polls taken in hepatology clinics, up to $31 \%$ of patients were taking alternative therapies. $^{28}$ These nontraditional medications should be inquired about and reviewed for safety. Vitamin A (betacarotene, retinol), a known hepatotoxin, is widely available over the counter. There are multiple reports of hepatic injury resulting from massive doses, usually doses greater than 100,000 IU per day. ${ }^{29}$ There are rare reports of vitamin A doses of as low as 25,000 IU per day leading to hepatic injury including sinusoidal fibrosis, steatosis, chronic hepatitis, and cirrhosis. Alcohol use is reported to potentiate the toxicity of vitamin A. ${ }^{29}$ Vitamin A has a dose-dependent toxicity and is safe to ingest in the normal diet. Patients with chronic liver disease should be warned about the risks of megadose vitamin A consumption. Other possible hepatotoxins available without prescription are niacin, Chinese herbal tea, Jin Bu Huan, germander, Kalms tablets, gentian, asafetida, valerian, mistletoe, senna fruit extracts, chaparral leaf, comfrey, bush herbal teas, and pennyroyal oil. ${ }^{29}$

Iron supplements, often in over-the-counter multivitamin preparations, are common and readily available to patients. In the usual state of health, excess dietary iron is not absorbed and is excreted in the stool. In the setting of many chronic liver diseases, there is a propensity for excess iron to accumulate in the hepatic parenchyma. $\mathrm{Pa}-$ tients with chronic hepatitis $\mathrm{C}$ and alcoholic liver disease have a tendency toward secondary hemosiderosis. Up to $30 \%$ of patients show evidence of excess serum iron accumulation by iron studies, and 10\% have liver tissue excesses. ${ }^{30,31}$ The mechanism for this iron overload state in chronic liver disease is unknown. Postulates include the following: release from injured hepatocytes with secondary uptake by Kupffer cells, acute-phase reactions associated with chronic inflammatory states, increased gastrointestinal absorption of iron, and ineffective erythropoeisis with redistribution from sites of utilization to sites of storage. ${ }^{31}$

Data indicating that iron overload is detrimental to those with chronic liver diseases have been accumulating. Several investigators have shown high hepatic iron concentration to be a predictor of nonresponse to interferon- $\alpha$ therapy in patients with chronic hepatitis C. ${ }^{32,33}$ In further support, when phlebotomy was used as the sole treatment in chronic hepatitis $\mathrm{C}$ in patients with histologically confirmed iron overload, there was a significant improvement in serum transaminase levels. ${ }^{34}$ A study from India showed that combining a low-iron diet with traditional treatment of hepatitis $\mathrm{C}$ increased response to treatment. ${ }^{35}$ Animal studies have demonstrated enhanced hepatotoxicity to alcohol in rat models when iron was coadministered. ${ }^{36}$

Iron induces hepatotoxicity through the increased generation of free radicals and increased peroxidation of lipids, which in turn lead to organelle dysfunction, lysosomal fragility, mitochondrial dysfunction, and ultimately cell death. ${ }^{36}$ Given these findings, patients with chronic liver diseases should avoid excessive iron intake. If a patient with chronic liver disease takes a multivitamin, it should not have iron as a component unless iron deficiency anemia is demonstrated. No evidence exists to suggest that normal dietary iron is harmful. Further randomized studies are needed to determine whether iron depletion in patients with evidence of tissue iron overload is beneficial in chronic liver disease.

\section{Dietary Fat}

Fatty infiltration of the liver is a basic response to a variety of injuries. The list of conditions that produce fatty infiltration is extensive and includes alcoholic liver disease, nonalcoholic steatohepatitis (NASH), hepatitis C, drug reactions, Wilson's disease, and other genetic diseases. ${ }^{37}$ Predisposing medical risk factors include diabetes mellitus, obesity, and elevated serum triglyceride levels. ${ }^{38}$ Fatty infiltration is often well tolerated with minimal to no inflammatory reaction. In some cases, however, an inflammatory reaction to the presence of fat can be intense, leading to elevated levels on liver injury tests and histologic changes of necroinflammation, fibrosis, and cirrhosis. ${ }^{39}$ A controlled trial by Ueno et al. demonstrated that a weight reduction program of diet and exercise can improve biochemical markers of hepatic injury and hepatic histology results in patients with NASH. ${ }^{40}$ Liver transaminase levels often return to normal with relatively minor weight reductions of 10 to 15 pounds. Deems et al. reported on a relation between liver injury test results and dietary intake. A study of dietary intake on 42 patients with chronic liver disease showed a correlation between high fat and oil consumption and elevated levels on liver injury tests. ${ }^{41}$ These observations suggest that a program of low-fat diet and exercise (supervised by a physician for appropriateness) would minimize hepatic steatosis. Gradual weight reduction should be recommended in patients with chronic liver disease and obesity.

\section{Preventive Measures in Cirrhosis}

Once cirrhosis is established, liver disease is considered irreversible. The Childs-Pugh score, which ranks severity on the basis of hepatic function tests and symptoms, 
has been shown to predict survival. ${ }^{4}$ Survival in those with early (Child's A) cirrhosis may be up to 15 to 20 years. Potential risks during this time interval include bleeding from esophageal varices and developing hepatocellular carcinoma. Preventive or early detection strategies have been evaluated for both of these conditions.

\section{$\beta$-Adrenergic Blockade in Prevention of First Variceal}

Bleed. Portal hypertension is a frequent complication of cirrhosis. When the portal pressures are consistently higher than $12 \mathrm{~mm} \mathrm{Hg}$, portal-systemic collaterals form. The rudimentary coronary vein dilates and leads to the formation of esophagogastric varices. Varices can be visualized in approximately $60 \%$ of patients with cirrhosis undergoing upper endoscopy. Patients with large varices will have bleeding episodes at a rate of approximately $40 \%$ per year. The first bleeding episode from varices may be associated with mortality rates as high as $50 \%{ }^{42}$

Endoscopic criteria have been defined to predict those patients who are at risk of variceal bleeding. These criteria include larger varices and red markings on variceal surface. ${ }^{43}$ The Child's score can then be applied to further predict those at risk. ${ }^{43}$ The ability to predict highrisk patients aids in targeting preventive strategies and allows those at low risk to avoid unnecessary medications. Several randomized controlled studies have shown that nonselective $\beta$-blockers (propranolol and nadolol) reduce the risk of initial variceal bleed in high-risk patients by about $50 \%(45 \%$ to $22 \%$ risk of bleeding over 2 years). ${ }^{44}$ The pulse is indicative of adequate dosing, and $\beta$-blockers are titrated to achieve a $25 \%$ reduction of the baseline pulse rate. Usual starting dose for propranolol is $10 \mathrm{mg}$ three times per day, but $\beta$-blockers are not uniformly tolerated. ${ }^{45}$ Isosorbide-5-mononitrate $(20 \mathrm{mg}$ twice daily) has been shown to be a good second-line agent when blockers are contraindicated or have adverse effects. ${ }^{46}$ If either no varices or small varices are found on initial endoscopy, medication prophylaxis may be withheld. Patients without varices on initial endoscopy should subsequently undergo surveillance endoscopy every 1 to 2 years to determine subsequent risk of bleeding by endoscopic criteria. ${ }^{43}$

Screening for Hepatocellular Cancer. Cirrhosis, with few exceptions, carries a high risk for the development of hepatocellular carcinoma HCC, a leading cause of death in a study of cirrhotic patients. ${ }^{47}$ Advanced HCC carries a poor prognosis, with TNM stage IV A tumors having a 3-year survival of $17 \% .{ }^{48}$ When HCC lesions are discovered early and are less than $2 \mathrm{~cm}$ at the time of resection, survival of up to $85 \%$ at 5 years has been reported. ${ }^{49}$ In patients with decompensated liver disease and a small HCC, successful liver transplantation with minimal recurrence has been reported. ${ }^{50}$

Two tests have been suggested for screening for HCC, the serum measurement of $\alpha$-fetoprotein (AFP) and liver ultrasonography. Both tests have significant false-positive and false-negative rates. When above $20 \mu \mathrm{g} / \mathrm{L}$, AFP measurement has a sensitivity of $64 \%$ and specificity of $91 \% .{ }^{50}$ Ultrasound has a reported sensitivity of $79 \%$ and specificity of $94 \%$ for the detection of HCC. Although imperfect when either test is used alone, multiple studies have confirmed the efficacy of AFP and ultrasound in combination for early detection. ${ }^{47,49}$ Some controversy still exists with regard to the frequency of testing required and achievement of cost-effectiveness. ${ }^{51}$ Because randomized trials in this patient group are difficult to perform, data to establish that early detection translates into better outcome are lacking. ${ }^{52}$ Although controversial, the existing data suggest a role for yearly ultrasounds and measurement of AFP every 6 months in patients with cirrhosis of the liver.

Prevention of Spontaneous Bacterial Peritonitis. The onset of infected ascitic fluid or SBP heralds a particularly advanced liver disease state. All patients with cirrhosis should be considered for liver transplantation once the diagnosis of ascites is made. ${ }^{53}$ In a randomized placebocontrolled study, norfloxacin was shown to reduce the risk of a second episode of SBP (after an initial episode) from $68 \%$ to $20 \% .{ }^{54}$ Enthusiasm for the use of norfoxacin is tempered by the development of resistant strains in subsequent cases of SBP with long-term use. This is particularly alarming before liver transplantation. ${ }^{55}$ Given these conflicting variables, a preventive strategy cannot be definitively recommended. If norfoxacin is to be used in selected patients, treatment should be limited to a 6 -month course to avoid resistance.

\section{Table 1. Patient Education Recommendations for Preventive Care in Chronic Liver Disease}

1. Avoid alcohol of all types (beer, wine, liquor, mixed drinks).

2. Receive vaccinations against hepatitis $A$ and $B$, if not already immune (your doctor can check blood work to determine this). Also receive a one-time pneumovax and yearly fall flu shots.

3. Avoid liver-toxic medications, such as over-the-counter pain killers (aspirin-like medications). Tylenol (acetaminophen) in less than 2 g per day is safest (one extra-strength every 6 hours). Review all other medications with your doctor.

4. Avoid iron supplementation unless your doctor has shown that you are iron deficient. (If you take a multivitamin, use a brand that does have iron as an ingredient.)

5. Eat a low-fat, "heart smart" diet; this is good for your liver as well as your heart.

6. Once cirrhosis develops, get screened yearly with upper endoscopy to follow your risk of bleeding and with ultrasound to detect liver cancer.

7. Inform all of your health care providers that you have liver disease. Consider wearing a medical alert bracelet. 


\section{CONCLUSIONS}

Many liver diseases have long natural histories, and there are few treatments that can directly alter their course. To maximize the time to cirrhosis, decrease the need for liver transplantation, and delay death, it becomes important to avoid other injury to the liver. The existing data show that the preventive strategies of alcohol avoidance, hepatitis vaccination, avoidance of NSAIDs, iron supplementation only when iron deficiency is demonstrated, and a low-fat diet are prudent in patients with chronic liver disease. Once cirrhosis develops, screening for hepatocellular cancer with AFP and ultrasound and screening for varices to reduce the risk of first variceal bleeding by treating with nonselective $\beta$-blockers may be appropriate. Prophylaxis against recurrent spontaneous bacterial peritonitis remains controversial. Recommendations that can be used for patient education are presented in Table 1.

\section{REFERENCES}

1. Alter JM, Margolis HS, Krawczynski K, et al. The natural history of community-acquired hepatitis $\mathrm{C}$ in the United States. N Engl J Med. 1992;327:1899-905.

2. Zein NN, Rakela J, Krawitt EL, et al. Hepatitis C virus genotypes in the United States: epidemiology, pathogenicity, and response to interferon therapy. Ann Intern Med. 1996;125:634-9.

3. Poupon RE, Balkau B, Eschwege E, et al. A multicenter, controlled trial of ursodial for the treatment of primary biliary cirrhosis. N Engl J Med. 1991;324:1548-54.

4. Propst A, Propst T, Zangerl G, et al. Prognosis and life expectancy in chronic liver disease. Dig Dis Sci. 1995;40:1805-15.

5. Thun MJ, Peto R, Lopez AD, et al. Alcohol consumption and mortality among middle-aged and elderly U.S. adults. N Engl J Med. 1997;337:1705-14.

6. Scheig R. Effects of ethanol on the liver. Am J Clin Nutr. 1970; 23:467-73.

7. Corrao G, Arico S. Independent and combined action of hepatitis $\mathrm{C}$ virus infection and alcohol consumption on the risk of symptomatic liver cirrhosis. Hepatology. 1998;27:914-9.

8. O'Conner PG, Schottenfeld RS. Patients with alcohol problems. N Engl J Med. 1998;592-601.

9. Wang JY, Lee SD, Tsai YT, et al. Fulminant hepatitis A in chronic HBV carrier. Dig Dis Sci. 1986;31:109-11.

10. Conteas C, Kao H, Rakela J, et al. Acute type A hepatitis in three patients with chronic HBV infection. Dig Dis Sci. 1983;28:684-6.

11. Keeffe EB. Is hepatitis A more severe in patients with chronic hepatitis B and other chronic liver diseases? Am J Gastroenterol. 1995;90:201-5

12. Akriviadis EA, Redeker AG. Fulminant hepatitis A in intravenous drug users with chronic liver disease. Ann Intern Med. 1989;110: 838-9.

13. Vento S, Garofano T, Renzini C, et al. Fulminant hepatitis associated with hepatitis A virus superinfection in patients with chronic hepatitis C. N Engl J Med. 1998;338:286-90.

14. Francis DP. The public's health unprotected: reversing a decade of underutilization of hepatitis B vaccine. JAMA. 1995;274:1242-3.

15. Bader TF. Hepatitis A vaccine. Am J Gastroenterol. 1996;91: 217-22.

16. Gardner P, Eickoff T, Poland GA, et al. Adult immunizations. Ann Intern Med. 1996;12:35-40.

17. Keeffe EB, Iwarson S, McMahon BJ, et al. Safety and immunoge- nicity of hepatitis A vaccine in patients with chronic liver disease. Hepatology. 1998;27:881-6.

18. Hess G, Clemens R, Bienzle U, et al. Immunogenicity and safety of an inactivated hepatitis A vaccine in anti-HIV positive and negative homosexual men. J Med Virol. 1995;46:40-2.

19. Andre FE. Summary of safety and efficacy data on a yeast-derived hepatitis vaccine. Am J Med. 1989;87(3A):14S-20S.

20. Hughes JM. Prevention of pneumococcal disease. MMWR. 1997;46:1-24.

21. Hughes JM. Prevention and control of influenza. MMWR. 1997;46:1-25.

22. Lee WM. Drug-induced hepatotoxicity. N Engl J Med. 1995;118-27.

23. Speeg KV, Bay MK. Prevention and treatment of drug-induced liver disease. Gastroenterol Clin North Am. 1995;24:1047-64.

24. Carson JL, Willet LR. Toxicity of nonsteroidal anti-inflammatory drugs: an overview of the epidemiological evidence. Drugs. 1993;46S:243-8.

25. Riley TR, Smith JP. Ibuprofen-induced hepatotoxicity in those with chronic hepatitis C: a case series. Am J Gastroenterol. 1998; 93:1563-5.

26. Rabinovitz M, Van Thiel DH. Hepatotoxicity of nonsteroidal antiinflammatory drugs. Am J Gastroenterol. 1992;87:1696-1704.

27. Zimmerman HJ, Maddrey WC. Acetaminophen hepatotoxicity with regular intake of alcohol: analysis of instances of therapeutic misadventure. Hepatology. 1995;22:767-73.

28. Flora K, Hahn M, Rosen H, et al. Milk thistle (Silybum marianum) for the therapy of liver disease. Am J Gastroenterol. 1998;93:139-43.

29. Bashir RM, Lewis JH. Hepatotoxicity of drugs used in the treatment of gastrointestinal disorders. Gastroenterol Clin North Am. 1995;24:937-67.

30. Riggio O, Montagnese F, Fiore P, et al. Iron overload in patients with chronic viral hepatitis: how common is it? Am $\mathrm{J}$ Gastroenterol. 1997;8:1298-1301.

31. Bisceglie AM, Axiotis CA, Hoofnagle JH, et al. Measurement of iron status in patients with chronic hepatitis. Gastroenterology. 1992;102:2108-13.

32. Olynyk JK, Reddy R, Di Bisceglie AM, et al. Hepatic iron concentration as a predictor of response to interferon alfa therapy in chronic hepatitis C. Gastroenterology. 1995;108:1104-9.

33. Van Thiel DH, Friedlander L, Fagiuoli S, et al. Response to interferon alpha therapy is influenced by the iron content of the liver. $J$ Hepatol. 1994;20:410-5.

34. Hayashi H, Takikawa T, Nishimura N, et al. Improvement of serum aminotransferase levels after phlebotomy in patients with chronic active hepatitis $\mathrm{C}$ and excess hepatic iron. Am J Gastroenterol. 1994;89:986-8.

35. Guptas RC, Mulhotra S, Lehandekar P, et al. Influence of low iron diet on the efficacy of interferon therapy in patients with chronic liver disease. Presented at the IXth Biennial Scientific Meeting Asian Pacific Association for the Study of the Liver, Kuala Lumpur, Malaysia, January 26-29, 1994.

36. Stal P, Hultcrantz R. Iron increases ethanol toxicity in rat liver. J Hepatol. 1993; 17:108-15.

37. Laurin J, Lindor KD, Crippin JS, et al. Ursodeoxycholic acid or clofibrate in the treatment of non-alcohol-induced steatohepatitis: a pilot study. Hepatology. 1996;23:1464-7.

38. Powell EE, Cooksley WG, Hanson R, et al. The natural history of nonalcoholic steatohepatitis: a follow-up study of forty-two patients for up to 21 years. Hepatology. 1990;11:74-80.

39. Bacon BR, Farahvash MJ, Janney CG, et al. Nonalcoholic steatohepatitis: an expanded clinical entity. Gastroenterology. 1994; 107:1103-9.

40. Ueno T, Sugawara H, Sujaku K, et al. Therapeutic effects of restricted diet in obese patients with fatty liver. J Hepatol. 1997;27:103-7.

41. Deems RO, Friedman LS, Friedman MI, et al. Relationship between liver biochemical tests and dietary intake in patients with liver disease. J Clin Gastroenterol. 1994;18:304-8. 
42. Kleber G, Hasan A, Sauerbruch T. Prophylaxis of first variceal bleeding. Bailliere Clin Gastroenterol. 1992;6:563-80.

43. DeFranchis R. Prediction of first variceal hemorrhage in patients with cirrhosis of the liver and esophageal varices. N Engl J Med. 1988;319:983-9.

44. Poynard T, Cales P, Pasta L, et al. Beta-adrenergic-antagonist drugs in the prevention of gastrointestinal bleeding in patients with cirrhosis and esophageal varices. N Engl J Med. 1991;324: 1532-8.

45. Grace N. Prevention of initial variceal hemorrhage. Gastroenterol Clin North Am. 1992;21:149-61.

46. Angelico M, Carli L, Piat C, et al. Isosorbide-5-mononitrate versus propranolol in the prevention of first bleeding in cirrhosis. Gastroenterology. 1993;104:1460-5.

47. Zoli M, Magalotti, D, Bianchi G, et al. Efficacy of a surveillance program for early detection of hepatocellular carcinoma. Cancer. 1996;78:977-85.

48. Iwatsuki S, Starzl TE, Sheahan DG, et al. Hepatic resection versus transplantation for hepatocellular carcinoma. Ann Surg. 1991; 214:221-9.
49. Maringhini A, Cottone M, Sciarrino E, et al. Ultrasonography and alpha-fetoprotein, in diagnosis of hepatocellular carcinoma in cirrhosis. Dig Dis Sci. 1988;33:47-51.

50. Mazzaferro V, Regalia E, Doci R, et al. Liver transplantation for the treatment of small hepatocellular carcinomas in patients with cirrhosis. N Engl J Med. 1996;334:693-9.

51. Durand F, Buffet C, Pelleetier G, et al. Screening of hepatocellular carcinoma in French patients. Dig Dis Sci. 1995;40:706-7.

52. Collier J, Sherman M. Screening for hepatocellular carcinoma. Hepatology. 1998;27:273-8.

53. Such J, Runyon BA. Spontaneous bacterial peritonitis. Clin Infect Dis. 1998;27:669-76.

54. Gines P, Rimola A, Planus R, et al. Norfloxacin prevents bacterial infection in cirrhosis: results of a double-blind, placebo controlled trial. Hepatology. 1990;12:716-24.

55. Campillo B, Dupeyron C, Richardet JP, et al. Epidemiology of severe hospital-acquired infections in patients with liver cirrhosis: effects of long term administration of norfloxacin. Clin Infect Dis. 1998;26:1066-70. 